\title{
T-3364366 Targets the Desaturase Domain of Delta-5 Desaturase with Nanomolar Potency and a Multihour Residence Time
}

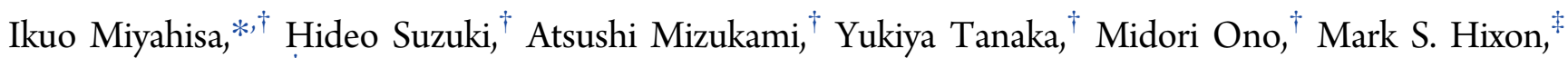
and Junji Matsui ${ }^{*} \dagger$

${ }^{\dagger}$ Pharmaceutical Research Division, Takeda Pharmaceutical Company Ltd., 26-1, Muraoka-Higashi 2-Chome, Fujisawa, Kanagawa 251-8555, Japan

${ }^{\ddagger}$ Takeda California Inc., 10410 Science Center Drive, San Diego, California 92121, United States

Supporting Information

ABSTRACT: Delta-5 desaturase (D5D) catalyzes the conversion from dihomo-gamma linoleic acid (DGLA) to arachidonic acid (AA). DGLA and AA are common precursors of anti- and pro-inflammatory eicosanoids, respectively, making D5D an attractive drug target for inflammatory-related diseases. Despite several reports on D5D inhibitors, their biochemical mechanisms of action (MOAs) remain poorly understood, primarily due to the difficulty in performing quantitative enzymatic analysis. Herein, we report a radio-

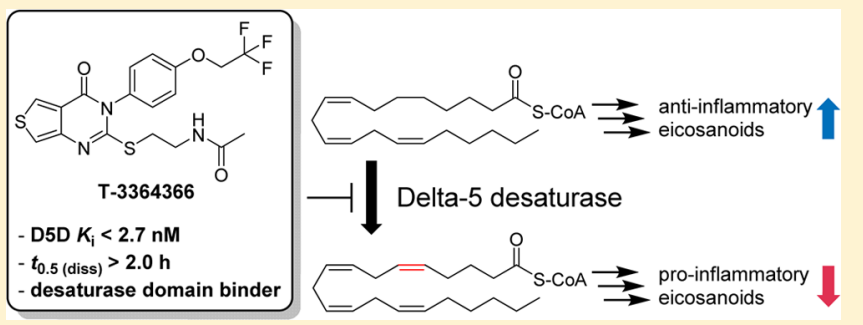
ligand binding assay to overcome this challenge and characterized T-3364366, a thienopyrimidinone D5D inhibitor, by use of the assay. T-3364366 is a reversible, slow-binding inhibitor with a dissociation half-life in excess of $2.0 \mathrm{~h}$. The long residence time was confirmed in cellular washout assays. Domain swapping experiments between D5D and D6D support $\left[{ }^{3} \mathrm{H}\right] \mathrm{T}-3364366$ binding to the desaturase domain of D5D. The present study is the first to demonstrate biochemical MOA of desaturase inhibitors, providing important insight into drug discovery of desaturase enzymes.

KEYWORDS: Delta-5 desaturase, T-3364366, slow-binding inhibition, mechanism of action (MOA)

$\mathrm{E}$ icosanoids are lipid signaling molecules synthesized by the oxidation of 20-carbon fatty acids and have numerous important biological functions as key regulators of inflammation. The eicosanoids derived from arachidonic acid (AA, C20:4 n-6) are generally pro-inflammatory substances, and their excess or uncontrolled production is implicated in a wide range of inflammation-related diseases from atherosclerosis to cancer. ${ }^{2,3}$ Thus, proteins involved in the biosynthesis and signal transduction of these eicosanoids have been intensively investigated as drug targets with significant attention of the pharmaceutical industry.

In vertebrates, $\mathrm{AA}$ is synthesized via a sequential enzymatic conversion from linoleic acid (LA, C18:2 n-6), which is an essential fatty acid. Desaturation of LA by delta- 6 desaturase (D6D) and subsequent $\mathrm{C}_{2}$ elongation by fatty acid elongase (Elovl5) yield dihomo-gamma linoleic acid (DGLA; 20:3, n-6). Next, delta-5 desaturase (D5D) introduces another double bond between $\mathrm{C}_{5}$ and $\mathrm{C}_{6}$ of DGLA, to produce AA (Scheme $1){ }^{5}$ The final oxidation catalyzed by $\mathrm{D} 5 \mathrm{D}$ is one of the ratecontributing steps in AA biosynthesis.

D5D is a member of the fatty acid desaturase family including D6D, delta-9 desaturase (stearoyl-CoA desaturase, SCD), and dihydroceramide desaturase 1 and 2. D5D catalyzes the $\mathrm{O}_{2}$-dependent dehydrogenation of dihomo-gamma linoleoyl-CoA to yield arachidonyl-CoA. ${ }^{6}$ DSD is an integral membrane protein localized in the endoplasmic reticulum and is expressed primarily in the liver and to a lesser degree in heart,
Scheme 1. Major Metabolic Pathway of Long-Chain Polyunsaturated Fatty Acids in Vertebrates

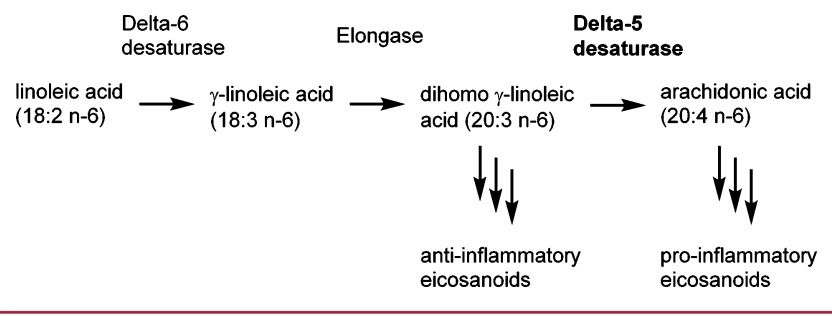

brain, and lung, although also found in other organs at low but detectable levels. ${ }^{7}$ D5D is a dual domain protein comprising a desaturase domain and a cytochrome $b 5$ domain. The desaturase domain catalyzes a desaturation reaction using a di-iron active center, while the cytochrome $b 5$ domain transfers electrons from $\mathrm{NADH}$ cytochrome $b 5$ reductase to the active center of the desaturase domain.

DGLA is a precursor of anti-inflammatory eicosanoids such as prostaglandin $\mathrm{E} 1\left(\mathrm{PGE}_{1}\right)$ and 15-hydroxytrienoic acid. ${ }^{8}$ Thus, inhibition of D5D is expected to exert dual antiinflammatory action, by decreasing AA-derived pro-inflammatory eicosanoids and simultaneously by increasing DGLA-

Received: June 23, 2016

Accepted: August 7, 2016

Published: August 10, 2016 
derived anti-inflammatory eicosanoids. In fact, D5D-deficient mice exhibited significant increase in $\mathrm{PGE}_{1}$ levels and decrease in $\mathrm{PGE}_{2}$ levels compared to wild-type mice, leading to antiinflammatory and antiproliferative cellular phenotypes. ${ }^{9}$ Taken together, inhibition of D5D by small molecules may attenuate inflammatory responses, potentially creating a next-generation anti-inflammatory agent. For example, Obukowicz et al. reported the discovery of several selective D5D inhibitors from compound screening, including CP-74006 (Chart 1) and other chemical classes of inhibitors (Supporting Information, Figure S1B). ${ }^{10}$ CP-74006 was further optimized to indazole series, displaying in vivo activity. ${ }^{11}$

Chart 1. Chemical Structures of CP-74006 and T-3364366<smiles>Nc1ccccc1C(=O)Nc1cccc(Cl)c1</smiles>

CP-74006
T-3364366<smiles>CC(=O)NCCSc1nc2cscc2c(=O)n1-c1ccc(OCC(F)(F)F)cc1</smiles>

Compound library screening using D5D enzymatic assay provided hits, which were then optimized leading to compounds such as T-3364366, a thienopyrimidinone D5D inhibitor (Chart 1). ${ }^{12}$ T-3364366 exhibited potent D5D inhibitory activity and excellent selectivity away from D6D and SCD in the enzymatic activity assay. In addition, T3364366 potently inhibits AA production in liver cell lines derived from human (HepG2) and rat (RLN-10) (Table 1). Curiously, the cellular $\mathrm{IC}_{50}$ values are more potent than that of enzymatic assay.

Table 1. IC $_{50}$ Values for Desaturase Activities of CP-74006 and $\mathrm{T}-3364366^{a}$

\begin{tabular}{lcc} 
& CP-74006 & T-3364366 \\
$\begin{array}{l}\text { enzymatic assay } \\
\text { D5D }(\mathrm{nM})\end{array}$ & $160(140-190)$ & $19(16-22)$ \\
D6D & $>10000$ & $6200(4100-9300)$ \\
SCD & $>10000$ & $>10000$ \\
cellular D5D assay (nM) & & $1.9(1.7-2.0)$ \\
HepG2 & $29(25-34)$ & $2.1(1.8-2.4)$ \\
RLN-10 & $26(22-30)$ & \\
${ }^{a}$ Numbers in parentheses represent & $95 \%$ & confidence intervals. \\
${ }^{b}$ Evaluated using rat liver microsomes. & \\
\hline
\end{tabular}

To elucidate the cause of this discrepancy, we initially performed a kinetic analysis of the inhibitor using an enzymatic assay of D5D. Unfortunately, the low sensitivity and assaylimited substrate concentration range failed to develop enzymatic assays for quantitative evaluations (Supporting Information, Supplementary Note S1).

For the lead generation effort in drug discovery, understanding the mechanism of biochemical interaction of a lead compound with its target is useful since it provides the basis for quantitative evaluation of the lead series. ${ }^{13}$ Although several D5D inhibitors have been reported, detailed biochemical and kinetic characterizations are lacking, resulting in poor understanding of the mechanisms of action (MOAs) of the inhibitors. Several challenges arise when examining D5D inhibitor potency. First, reported activity assays use HPLC or TLC for separation of D5D substrate and product, making the assays insensitive or low-throughput (or both). ${ }^{14,15}$ Second, D5D is an integral membrane protein and has yet to be purified to homogeneity, preventing crystallographic analysis and biochemical characterization with purified proteins, both of which have widely been utilized for the analysis of the soluble desaturases. ${ }^{16}$ Finally, strict control of active concentrations of substrates is difficult since one substrate is molecular oxygen and the other, a long-chain fatty acyl-CoA, forms micelles at concentrations around its $K_{\mathrm{m}}{ }^{17}$

To overcome the activity assay challenges, we directly analyzed the binding of T-3364366 by means of a radioligand binding assay using tritiated T-3364366 $\left(\left[{ }^{3} \mathrm{H}\right] \mathrm{T}-3364366\right)$ as a probe. Ligand binding assays using test compounds as probes offer an alternative strategy for direct observations of the kinetics and mechanisms of ligand binding in the absence of substrates. A filter binding assay was developed using rat liver microsomes D5D as a surrogate enzyme for the lower protein stability observed with recombinant human D5D (hD5D). Since T-3364366 exhibited similar inhibitory potencies in human and rat cellular assays (Table 1), the results obtained with rat liver microsomes should well reflect those with hD5D.

As an assay validation step, we performed a saturation binding experiment by varying the tritiated ligand concentrations. $\left[{ }^{3} \mathrm{H}\right] \mathrm{T}-3364366$ displayed specific and saturating binding to rat liver microsomes and exhibited single class of binding sites in the concentration range examined, consistent with 1:1 binding to a protein (Figure 1A). The specific binding

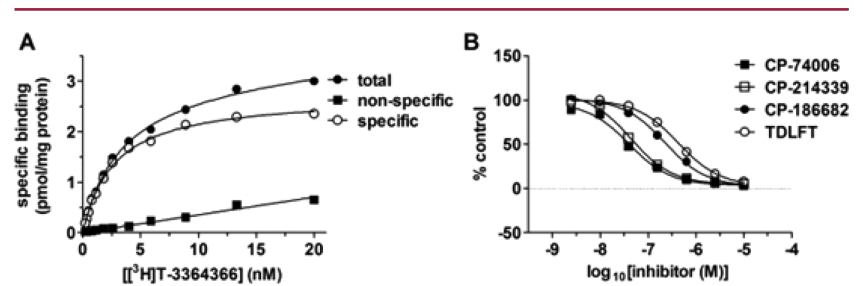

Figure 1. Development of a ligand binding assay using $\left[{ }^{3} \mathrm{H}\right] \mathrm{T}$ 3364366. (A) Saturation binding of $\left[{ }^{3} \mathrm{H}\right] \mathrm{T}-3364366$; specific binding (O) was calculated by subtracting nonspecific $(\boldsymbol{\square})$ from total binding (-). Data are presented as mean \pm standard errors of the mean (SEM; $n=3$ ). (B) Displacement of $\left[{ }^{3} \mathrm{H}\right] \mathrm{T}-3364366$ binding by known D5D inhibitors; DMSO and $10 \mu \mathrm{M}$ nonlabeled T-3364366 were used as $100 \%$ and $0 \%$ controls, respectively. Data are presented as mean \pm SEM $(n=3)$.

was fully displaced by CP-74006 and other selective D5D inhibitors (Figure 1B and Supporting Information, Figure S1A), but only partially displaced by the D6D inhibitor SC-26196 at elevated concentrations (Supporting Information, Figure S1B). ${ }^{10}$ The above study is consistent with the signal of $\left[{ }^{3} \mathrm{H}\right] \mathrm{T}-3364366$ reporting engagement of D5D rather than other proteins within the microsomal fraction including D6D. The apparent $2.7 \mathrm{nM}$ (2.5-2.9, 95\% confidence interval (CI)) $K_{\mathrm{d}}$ value after $150 \mathrm{~min}$ incubation agrees with the cellular assay $\mathrm{IC}_{50} \mathrm{~s}$ listed in Table 1, providing further validation of the ligand binding assay. The high sensitivity of this ligand displacement assay permits a 6 -fold reduction in microsome proteins over the enzymatic activity assay.

There are several possibilities that could cause the discrepancy in potency between enzymatic and cellular assay results, including compound activation and compound accumulation in cells in addition to overlooking timedependent inhibition. ${ }^{18}$ We first evaluated the slow onset of 
binding of T-3364366 using the binding assay. Various concentrations of $\left[{ }^{3} \mathrm{H}\right] \mathrm{T}-3364366$ were incubated with D5D, and specific binding at several time points was determined. Increase in the specific binding of $\left[{ }^{3} \mathrm{H}\right] \mathrm{T}-3364366$ was observed up to $2.5 \mathrm{~h}$, indicating time-dependent D5D binding of T-3364366 on the scale of our measurement (Figure 2A). In
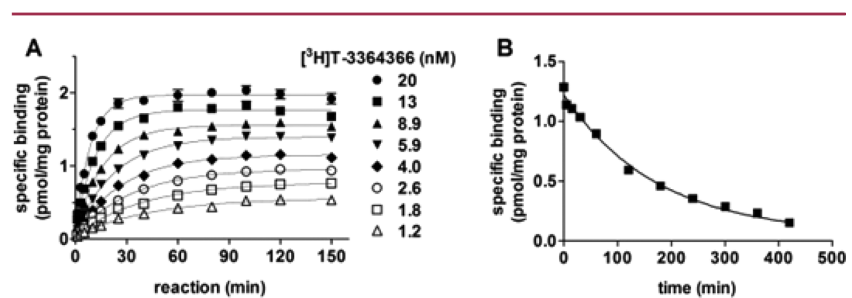

Figure 2. Slow-binding D5D inhibition of T-3364366. (A) Slow onset of binding kinetics of $\left[{ }^{3} \mathrm{H}\right] \mathrm{T}-3364366$; a single exponential association curve was fitted with data. Data are presented as mean \pm SEM $(n=6)$. (B) A dilution experiment of $\left[{ }^{3} \mathrm{H}\right] \mathrm{T}-3364366$; a single exponential decay curve was fitted to data. Data are presented as mean \pm SEM ( $n$ $=8)$.

contrast, after $2.5 \mathrm{~h}$ incubation, a much slower decrease in the specific binding was detected, which could be explained by D5D protein denaturation (Supporting Information, Figure S2). The slow decrease in specific binding could not be described by a simple mathematical function. Despite several attempts to build a mathematical model encompassing inhibitor association, dissociation, and loss of native D5D, we were unsuccessful in quantitatively determining the equilibrium and kinetic constants including the $K_{\mathrm{d}}$ value obtained above. Therefore, we report the upper limit of the true value (binding is tighter and dissociation slower than our model fit).

Time-dependent inhibition can arise from reversible inhibition with slow-binding kinetics or irreversible inhibition by covalent-bond formation. A reversibility test of T-3364366 was conducted by use of a dilution experiment. The binary complex between $\left[{ }^{3} \mathrm{H}\right] \mathrm{T}-3364366(6.0 \mathrm{nM})$ and D5D was produced by preincubation for $150 \mathrm{~min}$. Next, the mixture was diluted 2-fold with an excess amount of nonlabeled T-3364366 $(10 \mu \mathrm{M})$ to prevent rebinding of the dissociated ligand. The binding signal of $\left[{ }^{3} \mathrm{H}\right] \mathrm{T}-3364366$ slowly decreased after the dilution and was less than $10 \%$ of the control signal in $21 \mathrm{~h}$, suggesting reversible and slow-release interaction of T-3364366 (Supporting Information, Figure S3). As shown in Figure 2B, the dissociation half-life was determined from a dilution experiment as $2.0 \mathrm{~h}(1.9-2.1,95 \% \mathrm{CI})$ without considering the effect of the time-dependent loss of the specific binding.

To further exclude the possibility of covalent bond formation of T-3364366 with D5D, binding of $\left[{ }^{3} \mathrm{H}\right] \mathrm{T}-3364366$ after the addition of a protein denaturant was evaluated. Irreversible inhibitors through a covalent bond formation should remain attached to their targets even after the loss of the binding pocket by protein denaturation. Addition of final $2 \%$ trichloroacetate solution to D5D preincubated with $\left[{ }^{3} \mathrm{H}\right] \mathrm{T}$ 3364366 immediately abolished the specific binding of $\left[{ }^{3} \mathrm{H}\right] \mathrm{T}$ 3364366 , consistent with a noncovalent and reversible binding mechanism of T-3364366 (Supporting Information, Figure S4).

The dissociation half-life of T-3364366 is slow enough to expect duration of action in HepG2 assay after washout of the compounds in the medium. After treating CP-74006 and T3364366 with HepG2 cells for $3 \mathrm{~h}$, the inhibitors were removed from medium by washing cells three times and then the cells were pulsed with D5D substrate $\left[{ }^{14} \mathrm{C}\right]$ DGLA. Cells were then incubated for an additional $4 \mathrm{~h}$ and $\left[{ }^{14} \mathrm{C}\right] \mathrm{AA}$ production was monitored. While the washout produced nearly a thousand-fold shift inhibitory activity of AA production by CP-74006, it shifted the inhibitory activity by T-3364366 by only several fold (Figure 3). From the result, T-3364366 is expected to exert extended duration of action in vivo.
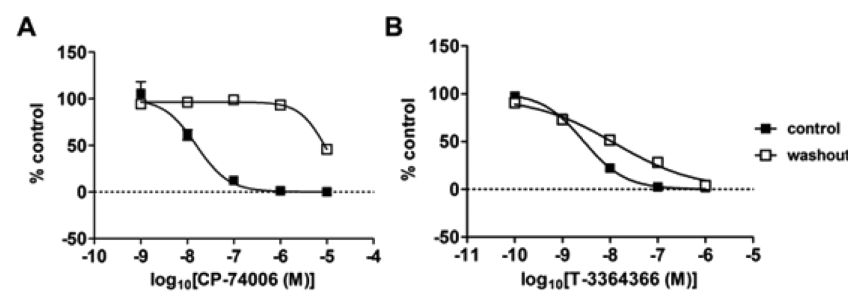

Figure 3. Duration of inhibition by CP-74006 (A) and T-3364366 (B) after washout in cellular D5D assays; normalized production of $\left[{ }^{14} \mathrm{C}\right] \mathrm{AA}$ from $\left[{ }^{14} \mathrm{C}\right]$ DGLA is plotted as a function of compound concentrations; DMSO and $10 \mu \mathrm{M}$ T-3364366 under no washout conditions were used as 100 and $0 \%$ controls, respectively. Data are presented as mean $\pm \operatorname{SEM}(n=4)$.

To consider the contributions of the slow off-rate of $\mathrm{T}$ 3364336 to in vivo efficacy, pharmacokinetic (PK) parameters of T-3364366 were determined (Table 2). T-3364366 exhibited

Table 2. Pharmacokinetic Parameters of T-3364366 ${ }^{a}$

$\begin{array}{lcccccc}\text { route } & \begin{array}{c}\text { dose } \\ (\mathrm{mg} / \mathrm{kg})\end{array} & \begin{array}{c}\mathrm{AUC}_{0-8 \mathrm{~h}} \\ (\mathrm{ng} \cdot \mathrm{h} / \mathrm{mL})\end{array} & \begin{array}{c}\mathrm{CL}_{\text {total }} \\ (\mathrm{L} / \mathrm{h} / \mathrm{kg})\end{array} & \begin{array}{c}V_{\text {dss }} \\ (\mathrm{L} / \mathrm{kg})\end{array} & \begin{array}{c}\mathrm{MRT} \\ (\mathrm{h})\end{array} & \begin{array}{c}F \\ (\%)\end{array} \\ \text { iv } & 0.10 & 65 & 1.5 & 1.1 & 0.65 & \\ \text { oral } & 1.0 & 260 & & & 1.8 & 41\end{array}$

${ }^{a}$ Mouse cassette dosing $(n=3)$; $F$ indicates bioavailability.

acceptable PK properties for oral administration in the mouse with its mean plasma residence time after intravenous administration $\left(\mathrm{MRT}_{\mathrm{iv}}\right.$ ) as $0.65 \mathrm{~h}$, which is shorter than the dissociation half-life of T-3364366.

Inhibitors with slow off-rates generally lead to extended durations of enzyme occupancy in vivo, even after drug concentrations decrease. ${ }^{19}$ The long residence time of $\mathrm{T}$ 3364366 could reduce potential off-target risks by decreasing systemic inhibitor exposure required for comparable in vivo efficacy.

Since D5D is a dual domain protein, several possibilities on MOA of D5D inhibition can be considered including inhibition of the desaturase reaction or perturbation of electron transduction. Thus, to determine the binding domain the inhibitor engages, we conducted a domain swapping experiment between D5D and D6D. These desaturases comprise desaturase and $b 5$ domains and share $76 \%$ amino acid sequence similarity. ${ }^{20}$ Since T-3364366 possesses excellent selectivity toward D6D, ligand binding assays against the domain-swapped mutants of desaturase and $b 5$ domain between D5D and D6D could reveal the binding domain of T-3364366. Based on the amino acid sequence alignment of the subtypes, we generated the domain-swapped mutants, D6D-b5 ${ }_{(1-100)} /$ D5D-des $(100-444)$ and D5D-b5 ${ }_{(1-99)} / \mathrm{D} 6 \mathrm{D}-\operatorname{des}_{(101-444)}$, and performed a $\left[{ }^{3} \mathrm{H}\right] \mathrm{T}$ 3364366 binding experiment against these recombinant proteins (Figure 4). $\left[{ }^{3} \mathrm{H}\right] \mathrm{T}-3364366$ only binds to the proteins with the D5D desaturase domain (wild-type D5D and D6D$\left.b 5_{(1-100)} / \mathrm{D} 5 \mathrm{D}-\operatorname{des}_{(100-444)}\right)$, consistent with T-3364366 inhibition of D5D through the interaction with the desaturase 


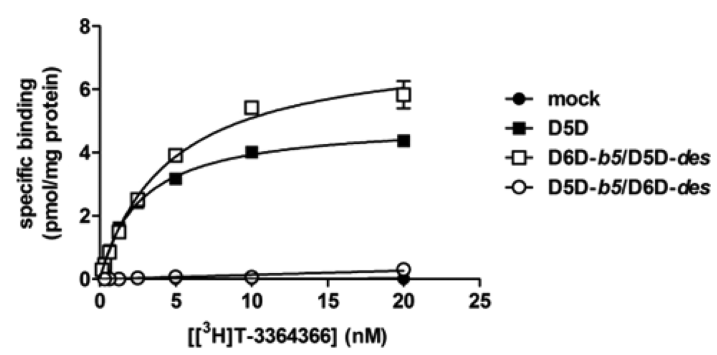

Figure 4. Identification of the $\left[{ }^{3} \mathrm{H}\right] \mathrm{T}-3364366$ binding site. Saturation binding experiments of $\left[{ }^{3} \mathrm{H}\right] \mathrm{T}-3364366$ were performed against mock (๑), wild-type D5D (ם), and the domain-swapped mutants, D6D-b5/ D5D-des ( $\square$ ) and D5D-65/D6D-des (O). Specific binding was determined as described in the materials and methods, Supporting Information. Data are presented as mean $\pm \operatorname{SEM}(n=3)$.

domain of the enzyme, but not with the electron transduction system.

To summarize, the MOA of T-3364366 was elucidated by developing a radioligand binding assay using the test compound as a probe. The advantages of the ligand binding assay over the reported enzymatic assays of D5D include sensitivity, throughput, and direct measurement of the binding, enabling the quantitative evaluation of T-3364366. Making the best use of these advantages, we demonstrate that T-3364366 exhibits slow-binding kinetics with its dissociation half-life of $>2.0 \mathrm{~h}$, which may in part explain the discrepancy between $\mathrm{IC}_{50}$ values in enzyme activity and cell-based assay. The difficulty in performing quantitative enzymatic assay for fatty acid desaturases may also lead to poor information on the MOA of inhibitors for enzymes of this family including D6D and SCD. Recently, a ligand displacement assay for SCD1 inhibitors using an azetidine compound $\left(\left[{ }^{3} \mathrm{H}\right] \mathrm{AZE}\right)$ was reported, but a detailed characterization of MOA of the compound has not yet reported. ${ }^{21}$ The biochemical analysis of $\left[{ }^{3} \mathrm{H}\right]$ AZE using a ligand binding method should be informative for further understanding of MOAs of inhibitors of desaturase family enzymes.

One of the important questions to be addressed on the biochemical MOA of T-3364366 is inhibition modality against a substrate, DGL-CoA. Since $\left[{ }^{3} \mathrm{H}\right] \mathrm{T}-3364366$ potently binds at least to a free form of the enzyme, we subsequently tried to investigate whether $\left[{ }^{3} \mathrm{H}\right] \mathrm{T}-3364366$ binds to the enzyme in the presence of DGL-CoA. Despite our attempt to perform the ligand binding assay, we failed to produce consistent results, presumably due to the micelle formation of the substrate (data not shown). In such cases, structural analysis of a target protein followed by mutational experiments can be one of the powerful solutions to determine the binding sites of substrates and inhibitors. Unfortunately, since no crystal structures of D5D have been reported to date, further chimeric or mutational experiments based on topological information on desaturase proteins, for example, may enable the speculation of binding sites and thus inhibition modality against the substrate. ${ }^{22}$

In conclusion, by use of the ligand binding assay, we demonstrate that T-3364366 is a potent, selective, and orally available D5D inhibitor, exhibiting a reversible, slow-binding interaction to the desaturase domain of D5D. T-3364366 is a promising lead compound with potent affinity and prolonged efficacy. The present study is the first to demonstrate MOA of a fatty acid desaturase inhibitor, providing important insight into drug discovery research on desaturase inhibitors.

\section{ASSOCIATED CONTENT}

\section{S Supporting Information}

The Supporting Information is available free of charge on the ACS Publications website at DOI: 10.1021/acsmedchemlett.6b00241.

Materials, synthetic procedure and analytical data for T3364366, full experimental procedures, and supporting figures (PDF)

\section{AUTHOR INFORMATION}

\section{Corresponding Authors}

*(I.M.) E-mail: ikuo.miyahisa@takeda.com. Phone: +81-46632-2783.

*(J.M.) E-mail: junji.matsui@takeda.com. Phone: +81-466-322780.

\section{Notes}

The authors declare no competing financial interest.

\section{ACKNOWLEDGMENTS}

We acknowledge all of the D5D project team members at Takeda, especially Shuichi Takagahara for preparation of rat liver microsomes, Nobuyuki Matsunaga for chemical synthesis of D5D inhibitors, and Masanori Nakakariya for analyzing PK data. We thank Tomoya Sameshima for helpful discussion on slow-binding kinetics.

\section{ABBREVIATIONS}

D5D, delta-5 desaturase; D6D, delta-6 desaturase; SCD, stearoyl-CoA desaturase; DGLA, dihomo-gamma linoleic acid; AA, arachidonic acid; LA, linoleic acid; MOA, mechanism of action; $\mathrm{NADH}$, nicotinamide adenine dinucleotide; PGE, prostaglandin E; DMSO, dimethyl sulfoxide; HPLC, highperformance liquid chromatography; TLC, thin-layer chromatography; PK, pharmacokinetics

\section{REFERENCES}

(1) Harizi, H.; Corcuff, J. B.; Gualde, N. Arachidonic-acid-derived eicosanoids: roles in biology and immunopathology. Trends Mol. Med. 2008, 14 (10), 461-469.

(2) Hansson, G. K.; Hermansson, A. The immune system in atherosclerosis. Nat. Immunol. 2011, 12 (3), 204-12.

(3) Wang, D.; Dubois, R. N. Eicosanoids and cancer. Nat. Rev. Cancer 2010, 10 (3), 181-193.

(4) Funk, C. D. Prostaglandins and leukotrienes: advances in eicosanoid biology. Science 2001, 294 (5548), 1871-1875.

(5) Nakamura, M. T.; Nara, T. Y. Structure, function, and dietary regulation of delta6, delta5, and delta9 desaturases. Annu. Rev. Nutr. 2004, 24, 345-376.

(6) Shanklin, J.; Cahoon, E. B. Desaturation and Related Modifications of Fatty Acids. Annu. Rev. Plant Physiol. Plant Mol. Biol. 1998, 49, 611-641.

(7) Cho, H. P.; Nakamura, M.; Clarke, S. D. Cloning, expression, and fatty acid regulation of the human delta-5 desaturase. J. Biol. Chem. 1999, 274 (52), 37335-37339.

(8) Wang, X.; Lin, H.; Gu, Y. Multiple roles of dihomo-gammalinolenic acid against proliferation diseases. Lipids Health Dis. 2012, 11, 25.

(9) Fan, Y. Y.; Monk, J. M.; Hou, T. Y.; Callway, E.; Vincent, L.; Weeks, B.; Yang, P.; Chapkin, R. S. Characterization of an arachidonic acid-deficient (Fads1 knockout) mouse model. J. Lipid Res. 2012, 53 (7), 1287-1295.

(10) Obukowicz, M. G.; Welsch, D. J.; Salsgiver, W. J.; MartinBerger, C. L.; Chinn, K. S.; Duffin, K. L.; Raz, A.; Needleman, P. Novel, selective delta6 or delta5 fatty acid desaturase inhibitors as 
antiinflammatory agents in mice. J. Pharmacol. Exp. Ther. 1998, 287 (1), 157-166.

(11) Baugh, S. D.; Pabba, P. K.; Barbosa, J.; Coulter, E.; Desai, U.; Gay, J. P.; Gopinathan, S.; Han, Q.; Hari, R.; Kimball, S. D.; Nguyen, H. V.; Ni, C. Y.; Powell, D. R.; Smith, A.; Terranova, K. M.; Wilson, A.; Yu, X. C.; Lombardo, V. K. Design, synthesis, and in vivo activity of novel inhibitors of delta-5 desaturase for the treatment of metabolic syndrome. Bioorg. Med. Chem. Lett. 2015, 25 (18), 3836-3839.

(12) Matsunaga, N.; Suzuki, H.; Asano, K.; Tokuhara, H.; Yamamoto, T.; Okamoto, R. Fused heterocyclic compound and application thereof. WO2012011591, 2012.

(13) Swinney, D. C. Molecular Mechanism of Action (MMoA) in Drug Discovery. Annu. Rep. Med. Chem. 2011, 46, 301-317.

(14) Zhang, L.; Ramtohul, Y.; Gagne, S.; Styhler, A.; Wang, H.; Guay, J.; Huang, Z. A multiplexed cell assay in HepG2 cells for the identification of delta-5, delta- 6 , and delta- 9 desaturase and elongase inhibitors. J. Biomol. Screening 2010, 15 (2), 169-176.

(15) Obukowicz, M. G.; Raz, A.; Pyla, P. D.; Rico, J. G.; Wendling, J. M.; Needleman, P. Identification and characterization of a novel delta6/delta5 fatty acid desaturase inhibitor as a potential antiinflammatory agent. Biochem. Pharmacol. 1998, 55 (7), 1045-1058.

(16) Moche, M.; Shanklin, J.; Ghoshal, A.; Lindqvist, Y. Azide and acetate complexes plus two iron-depleted crystal structures of the diiron enzyme delta9 stearoyl-acyl carrier protein desaturase. Implications for oxygen activation and catalytic intermediates. J. Biol. Chem. 2003, 278 (27), 25072-25080.

(17) Constantinides, P. P.; Steim, J. M. Physical properties of fatty acyl-CoA. Critical micelle concentrations and micellar size and shape. J. Biol. Chem. 1985, 260 (12), 7573-7580.

(18) Copeland, R. A. Evaluation of enzyme inhibitors in drug discovery. A guide for medicinal chemists and pharmacologists. Methods Biochem. Anal. 2005, 46, 1-265.

(19) Copeland, R. A.; Pompliano, D. L.; Meek, T. D. Drug-target residence time and its implications for lead optimization. Nat. Rev. Drug Discovery 2006, 5 (9), 730-739.

(20) Cho, H. P.; Nakamura, M. T.; Clarke, S. D. Cloning, expression, and nutritional regulation of the mammalian Delta-6 desaturase. J. Biol. Chem. 1999, 274 (1), 471-477.

(21) Tawa, P.; Falgueyret, J. P.; Guiral, S.; Isabel, E.; Powell, D. A.; Zuck, P.; Skorey, K. High-throughput scintillation proximity assay for stearoyl-CoA desaturase-1. J. Biomol. Screening 2011, 16 (5), 506-517. (22) Man, W. C.; Miyazaki, M.; Chu, K.; Ntambi, J. M. Membrane topology of mouse stearoyl-CoA desaturase 1. J. Biol. Chem. 2006, 281 (2), $1251-1260$. 